\title{
The impact of age on changes in quality of life among breast cancer survivors treated with breast-conserving surgery and radiotherapy
}

\author{
E J Bantema-Joppe ${ }^{1}, \mathrm{G} \mathrm{H}$ de Bock ${ }^{2}, \mathrm{M}$ Woltman-van lersel ${ }^{1}, \mathrm{D} \mathrm{M}$ Busz $^{1}, \mathrm{~A} \vee$ Ranchor $^{3}$, J A Langendijk ${ }^{1}$, \\ $\mathrm{J} \mathrm{H} \mathrm{Maduro*,1}^{*}$ and E R van den Heuvel ${ }^{2}$ \\ ${ }^{1}$ Department of Radiation Oncology, University of Groningen, University Medical Center Groningen, PO Box 30001,9700 RB \\ Groningen, The Netherlands; ${ }^{2}$ Department of Epidemiology, University of Groningen, University Medical Center Groningen, PO \\ Box 30 001, 9700 RB Groningen, The Netherlands and ${ }^{3}$ Department of Health Psychology, University of Groningen, University \\ Medical Center Groningen, PO Box 30 001, 9700 RB Groningen, The Netherlands
}

Background: The purpose of the study was to determine the impact of young age on health-related quality of life (HRQoL) by comparing HRQoL of younger and older breast cancer patients, corrected for confounding, and of young patients and a general Dutch population.

Methods: The population consisted of breast cancer survivors (stage 0-III) after breast-conserving surgery and radiotherapy. Health-related quality of life was prospectively assessed using the EORTC QLQ-C30 and QLQ-BR23 questionnaires. The association between age $(\leqslant 50 ; 51-70 ; \geqslant 70$ years) and HRQOL over time was analysed with mixed modelling. The clinical relevance of differences between/within age groups was estimated with Cohen's $D$ and consensus-based guidelines. The HRQoL data from the young patient cohort were compared with Dutch reference data at 3 years after radiotherapy.

Results: A total of 1420 patients completed 3200 questionnaires. Median follow-up was 34 (range 6-70) months. Median age was 59 (range 28-85) years. Compared with older subjects, young women reported worse HRQoL in the first year after radiotherapy, but clinical relevance was limited. Three years after radiotherapy, HRQoL values in the younger group were equal to those in the reference population. Pain and fatigue after radiotherapy improved, with medium clinical relevance.

Conclusions: Three years after radiotherapy for breast cancer, young age was not a risk factor for decreased HRQoL.

In the Netherlands, breast cancer is the most common malignancy in women with a yearly incidence of $\sim 14000$ cases and is rising (Netherlands Cancer Registry, 2014a,b). Early-stage breast cancer is treated with different modalities and may consist of surgery, radiotherapy and systemic treatment, including chemotherapy, endocrine treatment and targeted agents. The addition of these various treatment modalities improved treatment outcome significantly in terms of overall survival (Early Breast Cancer Trialists' Collaborative Group (EBCTCG) et al, 2011), with 5-year survival rates for stage I-II of $>92 \%$ (Bantema-Joppe et al, 2013; Netherlands Cancer Registry, 2014a,b).
Together with increasing breast cancer incidence, this improved outcome has resulted in a growing population of cancer survivors. The multimodality strategies may result in increased treatment-related side effects, ultimately leading to increased numbers of patients suffering from transient, persisting or even progressive late side effects (Gartner et al, 2009; Peuckmann et al, 2009; Hill-Kayser et al, 2011). In addition to the cancer diagnosis itself, these treatmentrelated side effects may have a major impact on patients' health-related quality of life (HRQoL) (Hau et al, 2013; Taghian et al, 2014).

*Correspondence: Dr JH Maduro; E-mail: j.h.maduro@umcg.nl

Received 22 August 2014; revised 13 November 2014; accepted 30 November 2014; published online 20 January 2015 
In the general non-cancer population, HRQoL depends on a number of factors, such as co-morbidities, sex, and age (Hjermstad et al, 1998; Schwarz and Hinz, 2001; Yun et al, 2007), the latter reflecting the normal process of ageing (Hjermstad et al, 1998). In cancer survivors, this normal process of ageing may influence adjustment to cancer and treatment over time. Thus, HRQoL measurements can be affected by age (Schroevers et al, 2004). Published data suggest that breast cancer has a greater impact on HRQoL in younger patients than in older ones (Schroevers et al, 2004; Howard-Anderson et al, 2012; Koch et al, 2013; Champion et al, 2014; Morrow et al, 2014) and a reduction in impact is seen with increasing age (Schroevers et al, 2006). However, other studies have not differentiated between the normal process of ageing and the impact of age on HRQoL. Therefore, in our study we made a direct comparison of age-related HRQoL outcome of Dutch breast cancer patients with reference data from the general Dutch population (van de Poll-Franse et al, 2011). Since young patients seem at higher risk for a large decrease in HRQoL after cancer treatment, we focussed specifically on this patient group.

In our prospective study, disease-free patients had been treated for early-stage breast cancer with breast-conserving surgery and radiotherapy were included. Our primary objective was to compare changes over time in various domains of HRQoL in breastconserved breast cancer patients between younger and older survivors, adjusted for confounding factors. Our secondary objective was to compare HRQoL in the younger patient cohort with the general population.

\section{MATERIALS AND METHODS}

Study population and data collection. The study population consisted of consecutive disease-free female patients treated for breast cancer (stage 0 -III) with postoperative radiotherapy after breast-conserving surgery, from January 2005 to March 2012 at the department of radiation oncology at the University Medical Center Groningen. Excluded were patients with a previous history of invasive cancer, except non-melanoma skin cancer or carcinoma in situ of the cervix; patients previously treated with radiotherapy to the thorax; patients diagnosed with synchronous contralateral breast cancer; patients treated with neo-adjuvant chemotherapy; patients with prophylactic ipsilateral mastectomy; and patients with insufficient proficiency in the Dutch language.

Since April 2008, all new breast-conserved patients treated at our department and all patients previously treated have been enrolled in a standard follow-up programme (SFP). Patients who were already in follow-up are seen yearly, and new patients are generally seen at 6 months after completion of radiotherapy and thereafter yearly, up to 5 years after radiotherapy.

In the SFP, data on quality of life and toxicity are prospectively collected, in addition to patient, tumour and treatment-related data. Furthermore, the National Cancer Institute (NCI) comorbidity index for breast cancer patients, modified according to Klabunde et al (2007), is used to assess co-morbidity. The SFP is used to monitor outcomes and is conducted under compliance of the hospital institutional review board regulations.

HRQoL questionnaires. During the routine follow-up visits at our department, patients were asked to complete the European Organisation for Research and Treatment of Cancer (EORTC) Quality of Life Questionnaire (QLQ)-C30 together with the EORTC QLQ-BR23 version 3.0 (Aaronson et al, 1993). The EORTC QLQ-C30 measures several dimensions in functioning and symptoms as well as global health status. The EORTC QLQ-BR23 focuses on systemic therapy side effects, arm and breast symptoms, and includes several sexual items.
Reference data (a representative sample of the normal Dutch population) were obtained from the Health and Health Complaints project from CentERdata. The EORTC QLQ-C30 and five additional questions on sexual items were available (van de PollFranse et al, 2011). Two sexual items were identical to the sexual functioning scale of the QLQ-BR23 module. We used reference data from young women ( $\leqslant 50$ years) $(N=380)$.

We focussed on scales that we considered to be specifically important to young women and on complaints commonly observed after radiotherapy. We used the following scales in our analysis: global health status, role functioning, emotional functioning, cognitive functioning, sexual functioning (all functional scales), fatigue, and pain (symptom scales). For functional scales, higher scores indicate better levels of functioning and/or better quality of life, while in symptom scales higher scores indicate more complaints.

Treatment. All patients were treated with post-operative threedimensional conformal radiotherapy with a simultaneous integrated photon boost (3D-CRT-SIB). The 3D-CRT-SIB was applied as described earlier (van der Laan et al, 2007). The fractionation schemes used were $28 \times 1.8 \mathrm{~Gy}$ to the whole breast, and with a simultaneous dose of 2.3 or 2.4 Gy to the tumour bed (total dose 64.4 or $67.2 \mathrm{~Gy}$ ). Regional radiotherapy, if indicated, consisted of photon radiotherapy of $28 \times 1.8$ Gy to the regional nodal areas.

Patients with node-positive disease and high-risk node-negative tumours were treated with adjuvant systemic therapy. Chemotherapy generally consisted of five cycles of 5-fluorouracil, epirubicine and cyclophosphamide (FEC) (90\% of the patients treated with chemotherapy). In total, $4.4 \%$ of the patients received taxane chemotherapy. In general, patients with positive lymph nodes were treated with chemotherapy before radiotherapy. In node-negative patients, radiotherapy was delivered after primary surgery and before the chemotherapy. Adjuvant endocrine treatment was indicated for all intermediate and high-risk hormonal receptorpositive patients. Depending on menopausal status, tamoxifen or aromatase inhibitors were given. Trastuzumab was added in patients receiving chemotherapy with tumours over-expressing the human epidermal growth factor receptor 2 (HER2).

Statistical analysis. Contingency tables were used to present the patient characteristics, stratified according to three age categories ( $\leqslant 50$ vs $51-70 v s \geqslant 70$ years at start of radiotherapy) with the chisquare test for comparison.

A generalised linear mixed model was used to determine the association between age and HRQoL over time, corrected for potential confounders. Tumour size ( $>2 \mathrm{~cm} v s \leqslant 2 \mathrm{~cm}$, based on the diameter of the primary tumour), pathologic nodal stage $(\mathrm{pN}+v s \mathrm{pN} 0)$, axillary clearance (yes $v s \mathrm{no})$, adjuvant systemic therapy (endocrine treatment $v s$ chemotherapy $v s$ both endocrine and chemotherapy $v s$ none), adjuvant trastuzumab (yes $v s$ no), regional radiotherapy (yes $v s$ no), and co-morbidity (medium/high vs low) were considered. Because of the high correlation (Pearson's $R>0.80$ ) between lymph-node status and axillary clearance, only axillary clearance was included in the model. The covariates without significant confounding effect were not included in the model. With this correction we were able to investigate the association between age and HRQoL over time independent of the other factors. A model was estimated for each scale separately. In the analyses of all end points, except for sexual functioning, the intercept and slope of follow-up time of patients (defined as time since completion of radiotherapy (continuous)) were considered to be random. For sexual functioning, only a random intercept was considered. Age category and the interaction with follow-up time, and all potential confounders were considered to be fixed effects. The interaction of age with follow-up time indicated if the change in HRQoL over time was different for the three age categories ( $\leqslant 50$ years was the reference category) and was tested with the 
likelihood ratio test. Note that this longitudinal data analysis fits patient-specific time profiles, takes into account the dependence between repeated observations on the same subject, and has the advantage of handling repeated data of subjects with varying numbers and unequally spaced follow-up times (Garson, 2012). In addition, the proposed models are typically suitable to analyze non-normally distributed response variables that are discrete and range from 0 to 100 .

The clinical relevance of the differences between young and older patients and the changes over time was estimated with the consensus-based guidelines of Cocks et al $(2011,2012)$ on the interpretation of EORTC QLQ-C30 scores (trivial, small, medium or large mean differences per scale) and by calculating Cohen's D. Cohen's D is an effect size used to compare differences between two means to the standard deviation of the whole population (Cohen, 1988). Effect sizes of $0.20-0.50$ are generally considered small, $0.50-0.80$ are moderate and clinically relevant, and 0.80 and above are considered a large effect and thus highly relevant. For the scales not included in the guidelines, changes were classified only by calculating Cohen's D. The agreement between the two classifications was good. The between-persons variation in our cohort was very large and much larger than the within-persons variation. Therefore, if available, we preferred to focus on the Cocks' classification.

To observe the changes in HRQoL over time, based on ageing in the reference population, the reference data from young subjects were analysed with a similar generalised linear model, including age in years and co-morbidity as covariates. For comparison, the NCI co-morbidity index was used as calculated in the breast cancer cohort. To test for mean differences in HRQoL between the prediction scores of young breast cancer patients and the young reference population at 3 years after completion of radiotherapy, a T-test was used. This time point was chosen because it was the longest follow-up with a reasonable number of questionnaires available. To address the problem of multiple testing, two-sided probability values of $<0.010$ were considered as statistically significant.

The statistical software SPSS (SPSS Inc, Chicago, IL, USA) for Windows, version 20.0, and SAS (Institute Inc, Cary, NC, USA), version 9.3 was used for statistical analysis.

\section{RESULTS}

Patient characteristics. During follow-up, 1548 patients were asked to complete the HRQoL questionnaires. In total, 1420 patients responded (overall compliance rate: 92\%) and 3200 completed questionnaires were available. Not all patients completed the 5-year follow-up; some had incomplete follow-up period and others were lost to follow-up. Consequently, different numbers of questionnaires were available per patient: 401 patients completed 1 questionnaire, 452 patients completed 2 questionnaires, 336 patients completed 3 questionnaires, and 231 patients completed 4 questionnaires. All questionnaires were included in the analysis.

During follow-up, the number of completed questionnaires decreased and missing data increased. In general, $<10 \%$ of the data were missing within the scales. Sexual functioning was an exception, with missing data ranging from $8 \%$ at 1 year to $29 \%$ at 5 years of follow-up (Supplementary Material).

Median follow-up time since completion of radiotherapy was 34 (range 6-70) months. At start of radiotherapy, the median age of all patients was 59 (range 28-85) years, and 292 patients (21\%) were 50 years or younger. Patients' characteristics, stratified for age, are summarised in Table 1 . Several factors were imbalanced between the three age groups. Young patients had significantly larger tumours, more lymph node positivity; consequently, they underwent axillary clearance more frequently and received adjuvant systemic therapy and trastuzumab more often. The older group patients $(\geqslant 70$ years) had more co-morbidities, although $74 \%$ had generally good health status, with low co-morbidity scores.

HRQoL functional scales. The crude end point measures with means and standard errors stratified for age over time are presented in the Supplementary Material (1. Functional scales and 2. Symptom scales).

The model of the HRQoL scales included the following confounders: axillary clearance, adjuvant systemic therapy, regional radiotherapy, and co-morbidity. By including these confounders, the predicted HRQoL outcomes of the breast cancer survivors are adjusted for these variables. The model thus predicts HRQoL in women treated without axillary clearance, without adjuvant systemic therapy, without regional radiotherapy, and with few co-morbidities after radiotherapy. Changes over time therefore represent only the effect of age. The lines in Figure 1 show the corrected HRQoL functional outcomes over time of the three age groups in the breast cancer cohort and the scores of the young reference population at one time point.

In general, HRQoL functional outcomes were good, with mean scores fluctuating around 90 points. Immediately after radiotherapy, the global health status of the three age groups was not significantly different (Table 2). Over time, the young age group showed a trend towards better global health, in contrast to the oldest group, which showed a trend towards worse global health $(P=0.004)$. However, none of the age groups showed significant or clinically relevant changes over time. At 3 years of follow-up, the young breast cancer patients had comparable global health to the reference population $(P=0.012)$.

Immediately after completion of radiotherapy, the young breast cancer survivors showed significantly worse HRQoL outcomes compared with older age groups on all other functional scales, except for sexual functioning. The outcomes for cognitive and emotional functioning in young women were worse than both the middle-aged and older women. With regard to role functioning, their scores were significantly worse than the middle-aged group but not the oldest group. However, based on Cohen's D and Cocks et al., these differences between the age groups were small or of trivial clinical relevance.

The development of role, emotional, and cognitive functioning over time of the oldest age group differed from the two younger age groups (role functioning $P<0.001$; emotional functioning $P=0.010$, and cognitive functioning $P<0.001$ ), with a trend towards better outcomes in the younger group and worse outcomes in the oldest age group. On the scores for role and emotional functioning, this positive trend over time increased significantly $(P<0.001)$. These improvements over time were of trivial clinical relevance in the middle-aged group. Although the significant improvement over time of the scores of the young women on these three scales was of small clinical relevance, at 3 years after radiotherapy this group had recovered to the levels in the young reference population (Figure 1B, role functioning; Figure 1C, emotional functioning; and Figure 1D, cognitive functioning).

The mean predicted scores of sexual functioning were worse than the other functional scales, with large and significant differences between the three age groups directly after radiotherapy $(P<0.001)$. Based on Cohen's D, the differences in sexual functioning between the young and oldest age group were moderate and clinically relevant. As in the reference population (data not shown), the mean sexual functioning was best in the youngest group, followed by the middle aged. Mean sexual functioning did not change over time in any of the age groups $(P>0.010)$. At 3 years after radiotherapy, the difference in sexual 
Table 1. Patient characteristics, stratified according to age $(n=1420)$

\begin{tabular}{|c|c|c|c|c|}
\hline Characteristic & $\leqslant 50$ years, $n=292$ & $51-70$ years, $n=895$ & $\geqslant 70$ years, $n=233$ & \\
\hline Median age (range) in years & $46(28-50)$ & $60(51-69)$ & $73(70-85)$ & \\
\hline & $n$ & $n$ & $n$ & $P$-value ${ }^{\text {a }}$ \\
\hline Pathologic T-stage & & & & $<0.001^{b}$ \\
\hline $\begin{array}{l}\text { PT in situ } \\
\text { pT1 } \\
\text { pT } \geqslant 2\end{array}$ & $\begin{array}{c}6(2.1 \%) \\
177(60.6 \%) \\
109(37.3 \%)\end{array}$ & $\begin{array}{c}31(3.5 \%) \\
667(74.5 \%) \\
197(22.0 \%)\end{array}$ & $\begin{array}{c}7(3.0) \\
175(75.1) \\
51(21.9)\end{array}$ & \\
\hline Pathologic N-stage & & & & $<0.001$ \\
\hline $\begin{array}{l}\mathrm{pNO} \\
\mathrm{pN}+ \\
\mathrm{pNx}\end{array}$ & $\begin{array}{c}182(62.3 \%) \\
104(35.6 \%) \\
6(2.1 \%)\end{array}$ & $\begin{array}{c}633(70.7 \%) \\
234(26.1 \%) \\
28(3.1 \%)\end{array}$ & $\begin{array}{c}188(80.7) \\
40(17.2) \\
5(2.1)\end{array}$ & \\
\hline Tumour size & & & & $<0.001$ \\
\hline $\begin{array}{l}\leqslant 2 \mathrm{~cm} \\
>2 \mathrm{~cm}\end{array}$ & $\begin{array}{l}181(62.2 \%) \\
110(37.8 \%)\end{array}$ & $\begin{array}{l}685(76.9 \%) \\
206(23.1 \%)\end{array}$ & $\begin{array}{r}180(77.3) \\
53(22.7)\end{array}$ & \\
\hline Axillary clearance & & & & $<0.001$ \\
\hline $\begin{array}{l}\text { No } \\
\text { Yes }\end{array}$ & $\begin{array}{l}180(61.6 \%) \\
112(38.4 \%)\end{array}$ & $\begin{array}{l}637(71.2 \%) \\
258(28.8 \%)\end{array}$ & $\begin{array}{r}190(81.5) \\
43(18.5)\end{array}$ & \\
\hline Adjuvant systemic therapy & & & & $<0.001$ \\
\hline $\begin{array}{l}\text { No } \\
\text { Endocrine treatment } \\
\text { Chemotherapy } \\
\text { Endocrine + chemotherapy }\end{array}$ & $\begin{array}{c}80(27.4 \%) \\
10(3.4 \%) \\
51(17.5 \%) \\
151(51.7 \%)\end{array}$ & $\begin{array}{l}401(44.8 \%) \\
175(19.6 \%) \\
100(11.2 \%) \\
219(24.5 \%)\end{array}$ & $\begin{aligned} 130 & (55.8) \\
96 & (41.2) \\
5 & (2.1) \\
2 & (0.9)\end{aligned}$ & \\
\hline Adjuvant trastuzumab & & & & 0.001 \\
\hline $\begin{array}{l}\text { No } \\
\text { Yes }\end{array}$ & $\begin{array}{c}268(91.8 \%) \\
24(8.2 \%)\end{array}$ & $\begin{array}{c}843(94.2 \%) \\
52(5.8 \%)\end{array}$ & $\begin{array}{l}231(99.1) \\
2(0.9)\end{array}$ & \\
\hline Regional radiotherapy & & & & 0.16 \\
\hline $\begin{array}{l}\text { No } \\
\text { Yes }\end{array}$ & $\begin{array}{c}269(92.1 \%) \\
23(7.9 \%)\end{array}$ & $\begin{array}{c}835(93.3 \%) \\
60(6.7 \%)\end{array}$ & $\begin{array}{l}224(96.1) \\
9(3.9)\end{array}$ & \\
\hline Co-morbidity & & & & $<0.001$ \\
\hline $\begin{array}{l}\text { Low } \\
\text { Medium/high }\end{array}$ & $\begin{array}{c}273(93.5 \%) \\
19(6.5 \%) \\
\end{array}$ & $\begin{array}{l}769(85.9 \%) \\
126(14.1 \%) \\
\end{array}$ & $\begin{array}{r}173(74.2) \\
60(25.8) \\
\end{array}$ & \\
\hline
\end{tabular}

functioning between the young breast cancer patients and the young reference population was not statistically significant $(P=0.044)$ (Figure 1E, sexual functioning).

The presence of co-morbidities negatively impacted mean outcomes of all functional scales. Role functioning was significantly worse in patients who had an axillary clearance $(P=0.006)$ or who were treated with the combination of chemotherapy and endocrine therapy compared with no systemic therapy $(P<0.001)$. Both the combination of chemotherapy and endocrine therapy and chemotherapy alone were associated with lower mean scores in cognitive functioning as compared with no systemic therapy $(P=0.001)$. Additional radiotherapy to the nodal areas was negatively associated with sexual functioning $(P=0.005)$ (data not shown).

HRQoL symptom scales. The mean predicted scores of the symptom scales are shown in Figure 2. In general, patients had few complaints, with mean scores of 15 points directly after radiotherapy. The young patients had significantly more fatigue and pain after radiotherapy, compared with the middle-aged patients $(P<0.001$ and $P=0.005)$, with limited clinical relevance. In all three age groups, a significant improvement over time was observed for the symptom scales. These improvements were largest in the youngest age group and of small (Cohen's D) to medium (Cocks et al.) clinical relevance, depending on which classification was used (Table 2). At 3 years after completion of radiotherapy, no significant differences were observed between the young patients and the young women from the reference population $(P>0.010)$.
Patients with co-morbidities had significantly more complaints of fatigue and pain, than those without co-morbidities $(P<0.001$ and $P<0.001)$. After an axillary clearance, patients reported significantly more pain $(P=0.001)$.

\section{DISCUSSION}

The results of our prospective cohort study indicate that in the first year after radiotherapy, several domains of HRQoL-such as role functioning, emotional functioning, cognitive functioning, fatigue, and pain-are more strongly affected in young breast-conserved cancer survivors than in older patients. At 3 years after radiotherapy, the patients appear to recover to the corresponding HRQoL values of young Dutch women from the general population. The changes over time on the symptom scales for pain and fatigue were clinically most relevant. Young patients had better sexual functioning compared with older patients, but no significant difference with the reference population.

Overall HRQoL. These findings are supported by previous studies. In two studies by Schroevers et al (2006, 2004), HRQoL was most affected in young survivors of several types of malignancies (50\% of the sample had breast cancer). The largest improvement in HRQoL was observed in the first year after diagnosis; after 8 years no significant differences in HRQoL were observed in the survivors of all ages compared with a reference population. 

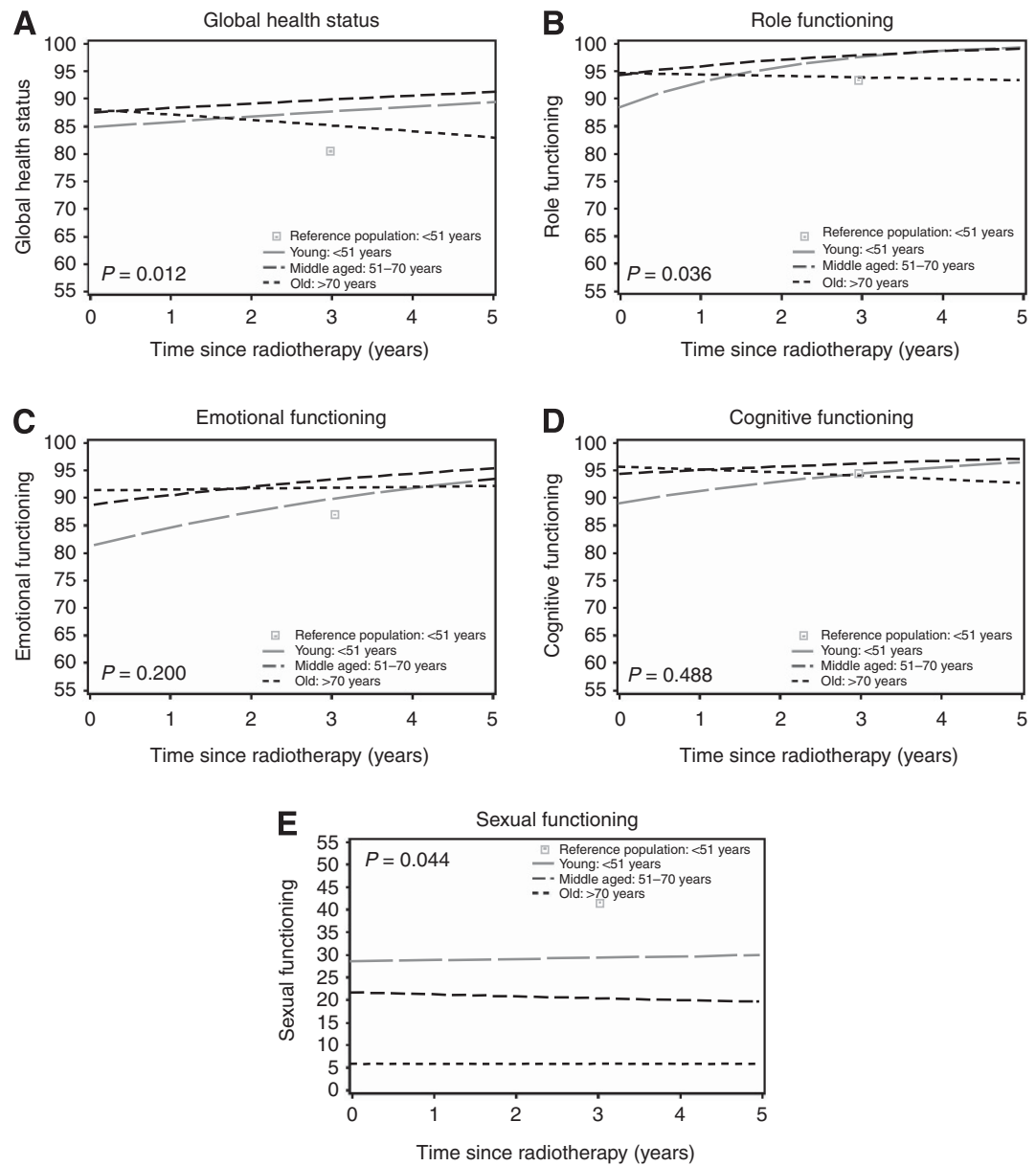

Figure 1. Predicted health-related quality of life of the functional scales of breast cancer survivors, compared with outcomes of a young Dutch reference population, stratified for age. Higher scores indicate better health-related quality of life. The lines represent outcomes in women treated without axillary clearance, without adjuvant systemic therapy, without regional radiotherapy, and with few co-morbidities present: (A) global health status, (B) role functioning, (C) emotional functioning, (D) cognitive functioning, and (E) sexual functioning. The outcomes of the young survivors are compared with the reference population at 3 years after completion of radiotherapy $(P$-value).

A systematic review on HRQoL and menopause-related symptoms in young breast cancer survivors showed that overall quality of life was compromised; mental functioning domains of HRQoL were affected more severely than physical functioning (Howard-Anderson et al, 2012). In our study, both the physical and the mental domains were impacted more in the youngest patient group immediately after completion of radiotherapy compared with older patients. However, our analysis showed that this finding has small clinical relevance.

Despite this worse HRQoL in young women immediately after radiotherapy, the improvements over time were also largest in this age group. On most functional scales, the changes were considered to be of small clinical relevance and in the symptom scales of medium relevance. Recently, Hsu et al (2013) observed improvements over time in many domains of HRQoL, assessed in longterm breast cancer survivors, with a relatively low mean age of 49.8 years at diagnosis, at both 1-year and 12-year post diagnosis. At 12 years after diagnosis, no clinically important differences were observed between breast cancer survivors and age-matched women without history of breast cancer in overall quality of life, role functioning, emotional functioning, and fatigue. The breast cancer patients reported even less pain than controls.

In several other studies on HRQoL in breast cancer patients, young age was a risk factor for poorer HRQoL and distress after treatment (Ganz et al, 2003; Engel et al, 2004; Hopwood et al, 2007; Hartl et al, 2010; Luutonen et al, 2011; Hau et al, 2013; Morrow et al, 2014). In our study, however, we found no differences in HRQoL between young breast cancer patients at 3 years after radiotherapy and an age-matched Dutch reference population. This difference in results is probably because we determined the independent effect of age by correcting for all other potential risk factors for decreased HRQoL, such as the administration of chemotherapy and endocrine treatment. The other studies did not make this correction.

The observed improvements over time in overall HRQoL, especially for young women, are probably the result of several mechanisms. First, a true improvement over time, with fewer physical complaints, may be present after the initial stress of diagnosis and first treatment phase. In young women, the observed recovery is larger than in older women. Young women have more physical flexibility and larger capacity to recover. In older women this capacity to recover is smaller, and is also influenced by the process of ageing itself. Second, and probably interacting with the first mechanism, the HRQoL measurements may be influenced by response shift. This means that the basis on which people make an HRQoL self-evaluation may also shift over time. However, the clinical significance of response shift is still unclear (Schwartz et al, 2006). One example of response shift can be observed our data on global health status, a scale on general quality of life. On this scale, young breast cancer survivors had at least comparable, and borderline significantly better HRQoL, compared with the reference population at 3 years of follow-up. 
Table 2. Predicted outcomes of the HRQoL scales, immediately after completion of RT and 3 years after completion of radiotherapy and the clinical relevance of the differences observed, stratified according to age ${ }^{a}$

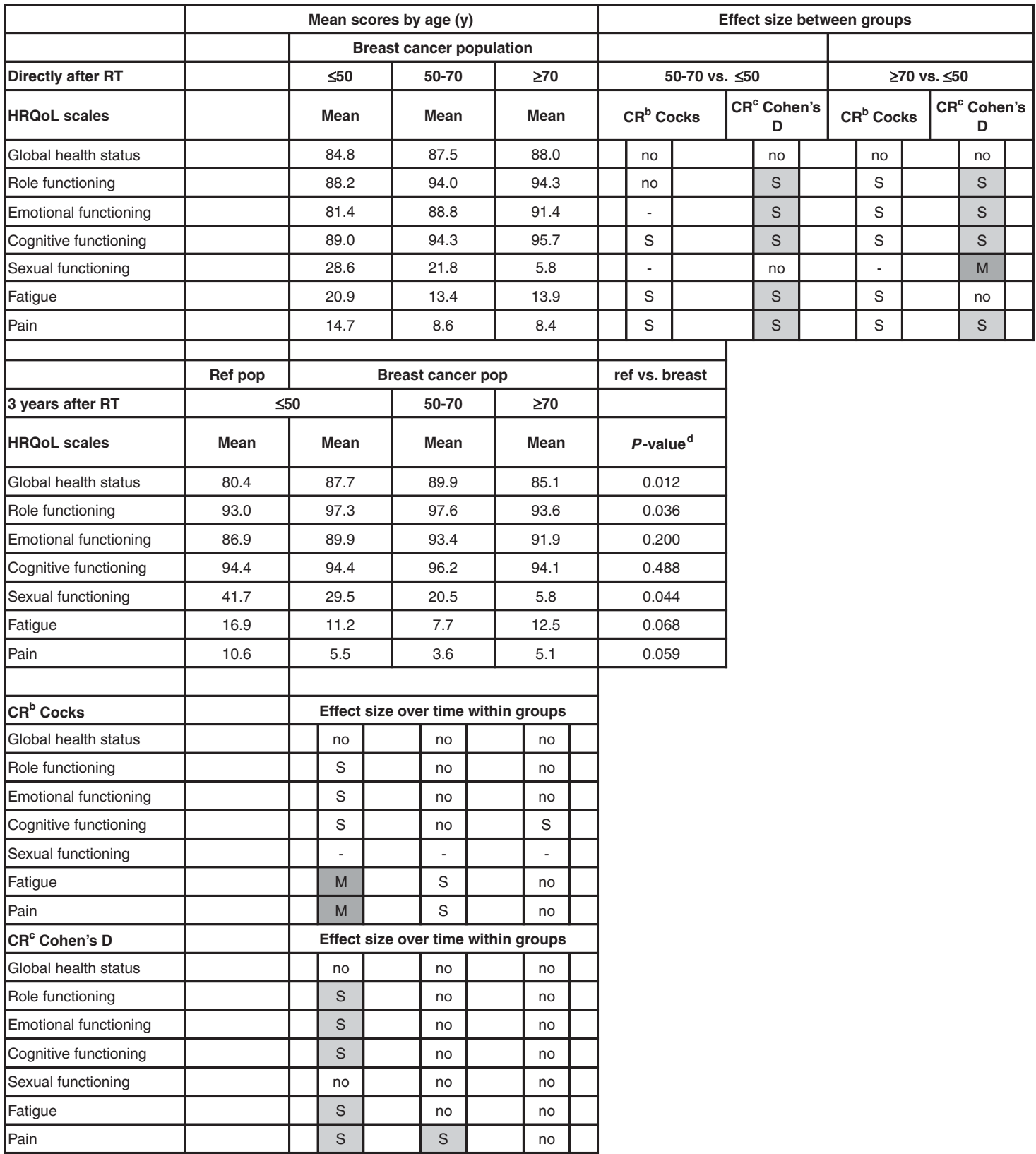

Abbreviations: $\mathrm{CR}=$ clinical relevance; $\mathrm{HRQOL}=$ health related quality of life; $\mathrm{RT}=$ radiotherapy $-=$ not available.

a Predicted scores for the specific subgroup of patients, treated with no axillary clearance, without adjuvant systemic therapy, without regional radiotherapy or co-morbidity.

${ }^{\mathbf{b}} \mathrm{CR}$ classification of the clinical relevance based on Cocks: 'no' ='trivial'; 'S'='small' (light grey); 'M' ='medium' (dark grey) differences.

${ }^{\mathrm{C}} \mathrm{CR}$ classification of the clinical relevance based on Cohen's D: 'no' = 'no clinical relevance'; 'S' = 'small' (light grey); 'M'= 'moderate' (dark grey) effect.

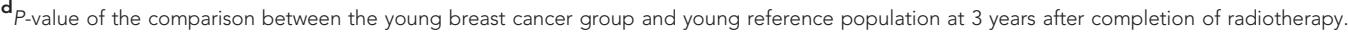

Pain and fatigue. On the symptom scales, fatigue and pain, which are commonly reported after radiotherapy, we observed a significant improvement over time in all age groups. Goldstein et al (2012) investigated cancer-related fatigue in 218 patients and concluded that fatigue after breast cancer is common, but generally selflimiting. In our larger cohort we confirmed this finding, which is opposite to what is generally perceived. Furthermore, based on multivariate analysis in an earlier study, Goldstein et al (2012) found that age was not a risk factor for fatigue after more than 6 months following adjuvant treatment. In contrast, we observed differences in fatigue between young and older patients after radiotherapy.

In previous research in 532 women treated with radiotherapy after breast-conserving surgery, we reported a higher incidence of pain in the chest wall in young patients at 1 year after radiotherapy ( $\leqslant 50$ years of age at diagnosis) compared with older women (Bantema-Joppe et al, 2012). This could be explained by the misattribution of pain and the decreased tendency to label a sensation as painful with increasing age (Gartner et al, 2009). Visser et al (2013) showed that response shift has a small, but unique contribution to the explanation of bodily pain after invasive surgery in cancer patients. Besides response shift, a true reduction in physical complaints may also be responsible for the observed improvements in pain over time.

Sexual functioning. Immediately after radiotherapy, sexual functioning differed significantly between the three age groups, with 

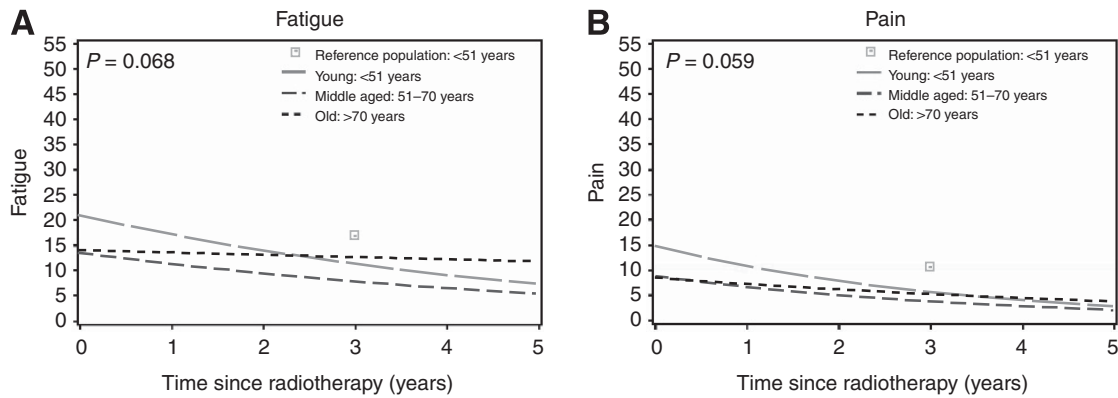

Figure 2. Predicted health-related quality of life of the symptom scales of breast cancer survivors, compared with outcomes of a young Dutch reference population, stratified for age. Higher scores indicate more complaints. The lines represent outcomes in women treated without axillary clearance, without adjuvant systemic therapy, without regional radiotherapy, and with few co-morbidities present: (A) fatigue and (B) pain. The outcomes of the young survivors are compared with the reference population at 3 years after completion of radiotherapy $(P$-value).

best sexual functioning in the youngest group and the worst in the oldest group. In contrast to nearly all other HRQoL domains, no changes over time in any age group were observed. The lack of change over time cannot be explained by the use of systemic treatment, resulting in vaginal dryness (Varras et al, 2003; Cella and Fallowfield, 2008) since we controlled for systemic treatment. The borderline insignificant difference in sexual functioning between the young breast cancer patients and the young Dutch women at 3 years might be attributed to the large amount of missing data. The relatively large proportion of missing data in the sexual functioning domain could have resulted in a lack of power to show differences. More specific research is needed on the effects of breast cancer treatment on sexual functioning.

Limitations. No data on socio-demographics and psychosocial factors were available for the breast cancer population. Healthrelated quality of life outcomes could have been influenced by these factors, thus limiting the generalisability of comparisons between the age groups of breast cancer patients and between the young breast cancer patients and the reference population.

Another limitation of our study was the number of missing questionnaires. In the statistical analysis, we assumed that the missing data were missing at random. Investigating the possible reasons for having missing data by Generalised Estimating Equations did not indicate otherwise. However, by including all patients, irrespective the number of questionnaires available, we could have biased the outcomes. First, patients without any health complaints were probably less likely to attend out-patient visits, leading to an underestimation of HRQoL. Second, patients with very serious non-cancer health problems might miss one or the rest of the follow-up visits, leading to an overestimation of the quality of life.

Longitudinal data in the reference population were also lacking. By matching the mean age and range of the reference group with the young breast cancer survivors at completion of radiotherapy, we were able to predict the HRQoL outcomes at that point in time. Age was then corrected by adding the follow-up time. By these means, we could compare and test for differences between the HRQoL of the young breast cancer cohort and the reference population, the latter reflecting normal ageing.

\section{CONCLUSION}

In our large prospective study, young women treated with radiotherapy after breast-conserving surgery for breast cancer have worse HRQoL in the first year after radiotherapy compared with their older counterparts, with small but significant clinical relevance. However, at 3 years after radiotherapy, they appear to recover to the values of the normal young Dutch population. These results indicate that on the medium long-term young age is not an independent risk factor for decreased HRQoL after radiotherapy in breast-conserved cancer patients.

\section{ACKNOWLEDGEMENTS}

We thank Lonneke van de Poll-Franse for supplying the data of the reference population.

\section{CONFLICT OF INTEREST}

The authors declare no conflict of interest.

\section{REFERENCES}

Aaronson NK, Ahmedzai S, Bergman B, Bullinger M, Cull A, Duez NJ, Filiberti A, Flechtner H, Fleishman SB, de Haes JC (1993) The European Organization for Research and Treatment of Cancer QLQ-C30: a quality-of-life instrument for use in international clinical trials in oncology. J Natl Cancer Inst 85: 365-376.

Bantema-Joppe EJ, Schilstra C, de Bock GH, Dolsma WV, Busz DM, Langendijk JA, Maduro JH (2012) Simultaneous integrated boost irradiation after breast-conserving surgery: physician-rated toxicity and cosmetic outcome at 30 months' follow-up. Int J Radiat Oncol Biol Phys 83: e471-e477.

Bantema-Joppe EJ, Vredeveld EJ, de Bock GH, Busz DM, Woltman-van Iersel M, Dolsma WV, van der Laan HP, Langendijk JA, Maduro JH (2013) Five year outcomes of hypofractionated simultaneous integrated boost irradiation in breast conserving therapy; patterns of recurrence. Radiother Oncol 108: 269-272.

Cella D, Fallowfield LJ (2008) Recognition and management of treatmentrelated side effects for breast cancer patients receiving adjuvant endocrine therapy. Breast Cancer Res Treat 107: 167-180.

Champion VL, Wagner LI, Monahan PO, Daggy J, Smith L, Cohee A, Ziner KW, Haase JE, Miller KD, Pradhan K, Unverzagt FW, Cella D, Ansari B, Sledge Jr GW (2014) Comparison of younger and older breast cancer survivors and age-matched controls on specific and overall quality of life domains. Cancer 120: 2237-2246.

Cocks K, King MT, Velikova G, de Castro Jr G, Martyn St-James M, Fayers PM, Brown JM (2012) Evidence-based guidelines for interpreting change scores for the European Organisation for the Research and Treatment of Cancer Quality of Life Questionnaire Core 30. Eur J Cancer 48: 1713-1721.

Cocks K, King MT, Velikova G, Martyn St-James M, Fayers PM, Brown JM (2011) Evidence-based guidelines for determination of sample size and interpretation of the European Organisation for the Research and Treatment of Cancer Quality of Life Questionnaire Core 30. J Clin Oncol 29: 89-96.

Cohen J (1988) Statistical Power Analysis for the Behavioral Sciences. Lawrence Erlbaum Associates: Hillsdale, NJ.

Early Breast Cancer Trialists' Collaborative Group (EBCTCG) Darby S, McGale P, Correa C, Taylor C, Arriagada R, Clarke M, Cutter D, Davies C, Ewertz M, Godwin J, Gray R, Pierce L, Whelan T, Wang Y, Peto R (2011) 
Effect of radiotherapy after breast-conserving surgery on 10-year recurrence and 15-year breast cancer death: meta-analysis of individual patient data for 10,801 women in 17 randomised trials. Lancet $\mathbf{3 7 8}$ $1707-1716$.

Engel J, Kerr J, Schlesinger-Raab A, Sauer H, Holzel D (2004) Quality of life following breast-conserving therapy or mastectomy: results of a 5-year prospective study. Breast J 10: 223-231.

Ganz PA, Greendale GA, Petersen L, Kahn B, Bower JE (2003) Breast cancer in younger women: reproductive and late health effects of treatment. J Clin Oncol 21: 4184-4193.

Garson GD (2012) Fundamentals of hierarchical linear and multilevel modeling, chapter 1. In Hierarchical Linear Modeling. Guide and Applications, Garson GD (ed.)pp 3-25. SAGE Publications, Inc: Thousand Oaks.

Gartner R, Jensen MB, Nielsen J, Ewertz M, Kroman N, Kehlet H (2009) Prevalence of and factors associated with persistent pain following breast cancer surgery. JAMA 302: 1985-1992.

Goldstein D, Bennett BK, Webber K, Boyle F, de Souza PL, Wilcken NR, Scott EM, Toppler R, Murie P, O’Malley L, McCourt J, Friedlander M, Hickie IB, Lloyd AR (2012) Cancer-related fatigue in women with breast cancer: outcomes of a 5-year prospective cohort study. J Clin Oncol 30: $1805-1812$.

Hartl K, Schennach R, Muller M, Engel J, Reinecker H, Sommer H, Friese K (2010) Quality of life, anxiety, and oncological factors: a follow-up study of breast cancer patients. Psychosomatics 51: 112-123.

Hau E, Browne L, Capp A, Delaney GP, Fox C, Kearsley JH, Millar E, Nasser EH, Papadatos G, Graham PH (2013) The impact of breast cosmetic and functional outcomes on quality of life: long-term results from the St. George and Wollongong randomized breast boost trial. Breast Cancer Res Treat 139: 115-123.

Hill-Kayser CE, Vachani C, Hampshire MK, Di Lullo GA, Metz JM (2011) Cosmetic outcomes and complications reported by patients having undergone breast-conserving treatment. Int J Radiat Oncol Biol Phys 83(3): 839-844.

Hjermstad MJ, Fayers PM, Bjordal K, Kaasa S (1998) Using reference data on quality of life-the importance of adjusting for age and gender, exemplified by the EORTC QLQ-C30 ( +3). Eur J Cancer 34: 1381-1389.

Hopwood P, Haviland J, Mills J, Sumo G, Bliss M (2007) The impact of age and clinical factors on quality of life in early breast cancer: an analysis of 2208 women recruited to the UK START Trial (Standardisation of Breast Radiotherapy Trial). Breast 16: 241-251.

Howard-Anderson J, Ganz PA, Bower JE, Stanton AL (2012) Quality of life, fertility concerns, and behavioral health outcomes in younger breast cancer survivors: a systematic review. J Natl Cancer Inst 104: 386-405.

Hsu T, Ennis M, Hood N, Graham M, Goodwin PJ (2013) Quality of life in long-term breast cancer survivors. J Clin Oncol 31: 3540-3548.

Klabunde CN, Legler JM, Warren JL, Baldwin LM, Schrag D (2007) A refined comorbidity measurement algorithm for claims-based studies of breast, prostate, colorectal, and lung cancer patients. Ann Epidemiol 17: 584-590.

Koch L, Jansen L, Herrmann A, Stegmaier C, Holleczek B, Singer S, Brenner H, Arndt V (2013) Quality of life in long-term breast cancer survivors a 10-year longitudinal population-based study. Acta Oncol 52: 1119-1128.

Luutonen S, Vahlberg T, Eloranta S, Hyvari H, Salminen E (2011) Breast cancer patients receiving postoperative radiotherapy: distress, depressive symptoms and unmet needs of psychosocial support. Radiother Oncol 100: 299-303.

Morrow PK, Broxson AC, Munsell MF, Basen-Enquist K, Rosenblum CK, Schover LR, Nguyen LH, Hsu L, Castillo L, Hahn KM, Litton JK, Kwiatkowski DN, Hortobagyi GN (2014) Effect of age and race on quality of life in young breast cancer survivors. Clin Breast Cancer 14: e21-e31.

Netherlands Cancer Registry. http://www.cijfersoverkanker.nl/selecties/ Dataset_1/img53f209789b792, accessed 18 August (2014a).

Netherlands Cancer Registry. http://www.cijfersoverkanker.nl/selecties/ dataset_2/img53f20a4a644c1, accessed 18 August (2014b).

Peuckmann V, Ekholm O, Sjogren P, Rasmussen NK, Christiansen P, Moller S, Groenvold M (2009) Health care utilisation and characteristics of long-term breast cancer survivors: nationwide survey in Denmark. Eur J Cancer 45: 625-633.

Schroevers M, Ranchor AV, Sanderman R (2006) Adjustment to cancer in the 8 years following diagnosis: a longitudinal study comparing cancer survivors with healthy individuals. Soc Sci Med 63: 598-610.

Schroevers MJ, Ranchor AV, Sanderman R (2004) The role of age at the onset of cancer in relation to survivors' long-term adjustment: a controlled comparison over an eight-year period. Psychooncology 13: 740-752.

Schwartz CE, Bode R, Repucci N, Becker J, Sprangers MA, Fayers PM (2006) The clinical significance of adaptation to changing health: a meta-analysis of response shift. Qual Life Res 15: 1533-1550.

Schwarz R, Hinz A (2001) Reference data for the quality of life questionnaire EORTC QLQ-C30 in the general German population. Eur J Cancer 37: 1345-1351.

Taghian NR, Miller CL, Jammallo LS, O’Toole J, Skolny MN (2014) Lymphedema following breast cancer treatment and impact on quality of life: a review. Crit Rev Oncol Hematol 92(3): 227-234.

van de Poll-Franse LV, Mols F, Gundy CM, Creutzberg CL, Nout RA, Verdonck-de Leeuw IM, Taphoorn MJ, Aaronson NK (2011) Normative data for the EORTC QLQ-C30 and EORTC-sexuality items in the general Dutch population. Eur J Cancer 47: 667-675.

van der Laan HP, Dolsma WV, Maduro JH, Korevaar EW, Hollander M, Langendijk JA (2007) Three-dimensional conformal simultaneously integrated boost technique for breast-conserving radiotherapy. Int $J$ Radiat Oncol Biol Phys 68: 1018-1023.

Varras M, Polyzos D, Akrivis C (2003) Effects of tamoxifen on the human female genital tract: review of the literature. Eur J Gynaecol Oncol 24: $258-268$.

Visser MR, Oort FJ, van Lanschot JJ, van der Velden J, Kloek JJ, Gouma DJ, Schwartz CE, Sprangers MA (2013) The role of recalibration response shift in explaining bodily pain in cancer patients undergoing invasive surgery: an empirical investigation of the Sprangers and Schwartz model. Psychooncology 22: 515-522.

Yun YH, Kim SH, Lee KM, Park SM, Kim YM (2007) Age, sex, and comorbidities were considered in comparing reference data for health-related quality of life in the general and cancer populations. J Clin Epidemiol 60: 1164-1175.

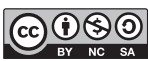

This work is licensed under the Creative Commons Attribution-NonCommercial-Share Alike 4.0 Unported License. To view a copy of this license, visit http://creativecommons. org/licenses/by-nc-sa/4.0/

Supplementary Information accompanies this paper on British Journal of Cancer website (http://www.nature.com/bjc) 